Revue internationale P.M.E.

Économie et gestion de la petite et moyenne entreprise

\title{
La succession dans les PME familiales : proposition d'un modèle de réussite du processus de désengagement du prédécesseur
}

\section{Louise Cadieux}

Volume 18, numéro 3-4, 2005

URI : https://id.erudit.org/iderudit/1008481ar

DOI : https://doi.org/10.7202/1008481ar

Aller au sommaire du numéro

Éditeur(s)

Presses de l’Université du Québec

ISSN

0776-5436 (imprimé)

1918-9699 (numérique)

Découvrir la revue

Citer cet article

Cadieux, L. (2005). La succession dans les PME familiales : proposition d'un modèle de réussite du processus de désengagement du prédécesseur. Revue internationale P.M.E., 18(3-4), 31-50. https://doi.org/10.7202/1008481ar
Résumé de l'article

Selon la littérature consultée dans deux champs connexes à celui de l'entreprise familiale, les deux dernières phases du processus de la succession pourraient correspondre à la période durant laquelle le prédécesseur se désengage de la gouvernance de son entreprise pour en laisser l'entière responsabilité à sa progéniture. Ne nous y étant jamais intéressés de manière spécifique, nous devons, par ailleurs, concéder ne pas comprendre cette période durant laquelle le prédécesseur réorganise plusieurs sphères de sa vie en même temps qu'il installe solidement sa relève. Cet article a donc pour objectif de présenter un modèle de réussite du processus de désengagement du prédécesseur élaboré à la suite d'une étude de cas menée auprès de cinq PME familiales ayant assuré leur premier transfert générationnel depuis au moins deux ans. 


\title{
La succession dans les PME familiales: proposition d'un modèle de réussite du processus de désengagement du prédécesseur
}

\author{
Louise CADIEUX \\ Département des sciences de la gestion \\ Université du Québec à Trois-Rivières
}

\section{MOTS CLÉS}

\section{Succession - PME familiales - Prédécesseur - Désengagement}

\begin{abstract}
RÉSUMÉ
Selon la littérature consultée dans deux champs connexes à celui de l'entreprise familiale, les deux dernières phases du processus de la succession pourraient correspondre à la période durant laquelle le prédécesseur se désengage de la gouvernance de son entreprise pour en laisser l'entière responsabilité à sa progéniture. Ne nous y étant jamais intéressés de manière spécifique, nous devons, par ailleurs, concéder ne pas comprendre cette période durant laquelle le prédécesseur réorganise plusieurs sphères de sa vie en même temps qu'il installe solidement sa relève. Cet article a donc pour objectif de présenter un modèle de réussite du processus de désengagement du prédécesseur élaboré à la suite d'une étude de cas menée auprès de cinq PME familiales ayant assuré leur premier transfert générationnel depuis au moins deux ans.
\end{abstract}

\section{L'AUTEURE}

LOUISE CADIEUX est professeure en management au Département des sciences de la gestion de l'Université du Québec à Trois-Rivières. Détentrice d'une maîtrise en gestion des PME, c'est dans le cadre de ses études doctorales, l'ayant mené à l'obtention d'un diplôme de DBA en administration des affaires qu'elle poursuit ses objectifs en faisant de la recherche sur les problématiques vécues en entreprise familiale, notamment sur celles liées à la transmission d'entreprises. Adresse: Département des sciences de la gestion, Université du Québec à Trois-Rivières, C.P. 500, Trois-Rivières, Québec, Canada, G9A 5H7. Téléphone: (819) 376-5011, poste 3114, télécopieur: (819)376-5079. Courriel:<louise_cadieux@uqtr.ca>. 


\begin{abstract}
In theory, the last two steps in the succession process - the joint management and withdrawal phases - differ from preceding phases in that they mark the successor's official entry into the family business as future head and the gradual retirement of the predecessor. Alone at the helm until that point, predecessors are faced with being the main instrument in setting in motion an important period of transition in their life where their position as leader is replaced by other roles that have yet to be clearly defined. The purpose of the current paper is to present a case study of five small and medium sized family businesses where the first generational transfer had been successfully completed and the predecessors had been officially retired for at least two years at the time of our study.
\end{abstract}

\title{
RESUMEN
}

Según la literatura consultada en dos campos conexos al de la empresa familiar, las dos últimas fases del proceso de sucesión podrían corresponder al período durante el cual el antecesor se desempeña de la gobernanza de su empresa para dejar toda responsabilidad a su descendiente. Nunca nos estamos interesado en ésto de manera específica, por eso debemos conceder no entender este período durante el cual el antecesor reorganiza varias esferas de su vida instalando un relevo sólido. Este artículo tiene como objetivo mostrar un modelo de éxito del proceso de desempeño del antecesor elaborado a continuación de un estudio de caso basado en cinco PyMEs familiares que han asegurado su primera transmisión generacional por lo menos desde hace dos años.

\section{ZUSAMMENFASSUNG}

Die massgebende Literatur im Gebiet der Familienunternehmen unterscheidet die beiden letzten Phasen der Nachfolgeplanung in eine Periode, in welcher der Vorgänger sein Engagement bei der Unternehmensführung reduziert und anschliessend die volle Verantwortung an seinem Nachwuchs übergibt. Ohne uns jemals speziell für diesen Prozess interessiert zu haben, müssen wir zugestehen, dass wir diese Periode, in der ein Unternehmer die verschiedensten Bereiche seines Lebens neu organisiert und dabei auch noch einen Nachfolger im Betrieb einführt, in den Einzelheiten nicht verstehen. Der vorliegende Artikel hat daher zum Ziel ein Prozessmodell vorzustellen, das eine erfolgreiche Nachfolgeplanung und -einführung umschreibt. Das beschriebene Modell basiert auf fünf Fallstudien aus Familienunternehmen, die den ersten Generationenwechsel seit mindestens zwei Jahren vollziehen.

\section{Introduction}

Plusieurs PME ayant vu le jour après la Seconde Guerre mondiale (Allouche et Amann, 1998; Julien, 2000) sont aujourd'hui confrontées au même défi, celui des transferts de la direction et de la propriété à la nouvelle génération. Sont-elles prêtes à relever un tel défi? Il semble que non puisque les résultats de récentes enquêtes effectuées auprès de dirigeants d'entreprises familiales révèlent que,

Revue internationale P.M.E., vol. 18, $\mathrm{n}^{\text {os }} 3-4,2005$

(C) 2005 - Presses de l'Université du Québec

Édifice Le Delta I, 2875, boul. Laurier, bureau 450, Sainte-Foy, Québec G1V 2M2 • Tél.: (418) 657-4399 - www.puq.ca

Tiré de: Revue internationale P.M.E., vol. 18, nos 3-4, sous la direction de Louis Raymond • PME1803N

Tous droits de reproduction, de traduction et d'adaptation réservés 
même si plus de la moitié prévoient se retirer avant l'an 2010, peu ont établi un plan ou même choisi un successeur (Cromie et al., 2001 ; FFI, 2003). Selon la littérature consultée, cela pourrait en grande partie s'expliquer par la difficulté qu'a le prédécesseur à se distancier de la gouvernance d'une entreprise dans laquelle il a souvent tout investi (Barnes et Hershon, 1976; Christensen, 1979; Kets de Vries, 1993 ; Pailot, 2000; Peay et Dyer, 1989; Sonnenfeld, 1988). Toute une génération d'entreprises serait-elle donc condamnée? Bien sûr que non, car au moins le tiers réussissent à assurer le passage de leur entreprise à la seconde génération (FFI, 2003)! Mais que se passe-t-il lorsque le prédécesseur réussit à faire ce qui semble si difficile pour d'autres? Comment lâche-t-il prise? Le fait-il abruptement? Ou, au contraire, prend-il son temps? A-t-il des périodes de doute? Ou de remises en question? Et comment occupe-t-il son temps après s'être retiré de la gouvernance de son entreprise? Les recherches empiriques consultées ne nous permettant pas de répondre clairement à ces questions, cet article a pour objectif de présenter une étude de cas menée auprès de cinq PME familiales ayant réussi leur premier transfert générationnel et dans lesquelles les prédécesseurs étaient, au moment de l'étude, officiellement retirés de la gouvernance depuis au moins deux ans. Pour nous, mieux comprendre ce qu'il en est du processus de désengagement du prédécesseur pourra certes être utile pour quiconque, qu'il s'agisse des prédécesseurs, des successeurs, des membres de leur famille ou des intervenants devant prodiguer de leur mieux support et conseils. Toutefois, avant d'attaquer le cœur du sujet auquel nous nous intéressons, nous allons faire un bref retour sur le processus de la succession.

\section{Le processus de la succession: une vue d'ensemble}

Dans la littérature recensée sur le processus de la succession en entreprise familiale, nous pouvons distinguer deux principaux types de modèles. Alors que les premiers décrivent les étapes des cycles de vie des organisations (Barnes et Hershon, 1976; Gersick et al., 1997; Holland et Boulton, 1984; Holland et Oliver, 1992; Murray, 2003; Peiser et Wooten, 1983), les seconds s'intéressent plutôt à l'interaction existant entre le prédécesseur et le successeur tout au long du processus (Churchill et Hatten, 1987; Handler, 1990; Hugron, 1992; Longenecker et Schoen, 1978). Développés principalement par le biais d'études de cas, les modèles consultés nous permettent de comprendre combien la succession est un processus durant lequel les rôles du prédécesseur et du successeur évoluent de manière dépendante et imbriquée, dans le but ultime de transférer la direction et la propriété de l'entreprise à au moins un membre de la prochaine génération. Partant du principe que le processus débute dès le moment où le prédécesseur fonde son entreprise et se termine lors de son retrait définitif, les modèles retenus peuvent être résumés par les quatre phases suivantes: l'initiation, l'intégration, le règne conjoint et le désengagement.

Revue internationale P.M.E., vol. 18, $\mathrm{n}^{\text {os }} 3-4,2005$

(C) 2005 - Presses de l'Université du Québec

Édifice Le Delta I, 2875, boul. Laurier, bureau 450, Sainte-Foy, Québec G1V 2M2 • Tél.: (418) 657-4399 - www.puq.ca

Tiré de: Revue internationale P.M.E., vol. 18, nos $3-4$, sous la direction de Louis Raymond - PME1803N

Tous droits de reproduction, de traduction et d'adaptation réservés 
Durant la première phase, seul maître à bord, le prédécesseur voit à la gestion courante et complète de l'entreprise. Même si le successeur n'y a pas encore de place apparente, c'est alors qu'il est initié au fonctionnement de l'entreprise (Churchill et Hatten, 1987; Longenecker et Schoen, 1978; Gersick et al., 1997; Handler, 1990; Hugron, 1992) et qu'il peut développer une perception positive de son parent comme dirigeant (Matthews, Moore et Fialko, 1999). La deuxième phase débute lorsque le successeur intègre l'entreprise. Y occupant souvent un poste à temps partiel ou saisonnier, c'est durant cette période qu'il se familiarise avec plusieurs aspects de l'environnement interne de l'organisation et développe une bonne part de ses compétences techniques (Churchill et Hatten, 1987; Handler, 1990; Longenecker et Schoen, 1978). La troisième phase, celle du règne conjoint, se démarque par l'entrée officielle du successeur à ce titre dans l'entreprise familiale (Stavrou, 1998). Dès lors, le prédécesseur et le successeur travaillent ensemble dans le but de transférer pouvoirs et responsabilités (Handler, 1990; Holland et Oliver, 1992), et cela, jusqu'à ce que ce dernier devienne autonome dans les décisions concernant l'entreprise (Churchill et Hatten, 1987; Longenecker et Schoen, 1978). Enfin, la dernière phase du processus est caractérisée par le retrait officiel du prédécesseur et le transfert complet des responsabilités (Churchill et Hatten, 1987; Hugron, 1992), du leadership, de l'autorité (Handler, 1990) et, dans certains cas, de la propriété (Barnes et Hershon, 1976; Churchill et Hatten, 1987; Hugron, 1992).

Comme nous venons de le voir, des quatre phases du processus de la succession, les deux dernières se singularisent par l'introduction officielle du successeur dans l'entreprise de même que par le retrait progressif du prédécesseur. Or, selon Handler (1994), c'est durant cette période que le prédécesseur est le plus susceptible de faire avorter le processus. Dans ce cas, le successeur n'assumera jamais le rôle de dirigeant lui étant réservé tant et aussi longtemps que le prédécesseur restera en poste. Selon la littérature consultée, deux principales raisons peuvent expliquer la difficulté du prédécesseur à définitivement lâcher prise. Profondément liées aux caractéristiques habituellement attribuées aux entrepreneurs, la première renvoie au degré d'identification et au lien d'attachement que le prédécesseur a développés avec son entreprise, tandis que la seconde concerne ses craintes face à son éventuel retrait du monde des affaires (Barnes et Hershon, 1976; Christensen, 1979; Kets de Vries, 1993; Pailot, 2000; Peay et Dyer, 1989; Sonnenfeld, 1988). Mais, en matière de retraite, les dirigeants d'entreprises familiales sont-ils vraiment des êtres d'exception? Il faut croire que non puisque lorsque Forteza et Prieto (1994) ont interrogé des futurs retraités occupant divers types de postes, plus des deux tiers avouaient avoir une perception négative de la retraite. Selon les répondants, avant même de passer à l'acte, prendre leur retraite signifiait amorcer une période de leur vie durant laquelle il leur serait quasiment impossible de se réaliser et/ou de se faire reconnaître.

Revue internationale P.M.E., vol. 18, $\mathrm{n}^{\text {os }} 3-4,2005$

(C) 2005 - Presses de l'Université du Québec

Édifice Le Delta I, 2875, boul. Laurier, bureau 450, Sainte-Foy, Québec G1V 2M2 - Tél.: (418) 657-4399 - www.puq.ca

Tiré de: Revue internationale P.M.E., vol. 18, nos $3-4$, sous la direction de Louis Raymond - PME1803N

Tous droits de reproduction, de traduction et d'adaptation réservés 
Pour le prédécesseur, assurer la pérennité de son entreprise comporte des défis de taille, dont le principal est celui de passer les rênes à son successeur tout en faisant face à de nouvelles réalités touchant plusieurs sphères de sa vie. Qu'il s'agisse des activités de planification ou du processus de la succession en tant que tel, les résultats des études empiriques consultées convergent. La continuité des entreprises familiales dépend grandement de la capacité du prédécesseur à se désengager de la gouvernance de son entreprise et d'en laisser l'entière responsabilité à son successeur (Barnes et Hershon, 1976; Christensen, 1979; Kets de Vries, 1993; Pailot, 2000; Peay et Dyer, 1989; Sonnenfeld, 1988). Or, s'étant jusqu'à ce jour peu intéressés à ce que font ceux qui réussissent ce passage difficile, nous devons admettre ne pas réellement comprendre «comment, dans des cas de PME familiales ayant réussi le passage de la première à la deuxième génération, le prédécesseur se désengage de son entreprise».

\section{Le processus de désengagement du prédécesseur: le contexte théorique}

Pour nous, comprendre comment le prédécesseur se désengage de son entreprise fait appel à deux champs de recherche jusqu'ici peu exploités dans la littérature sur la succession dans les entreprises familiales, soit ceux de la retraite et de la transition de rôle. C'est donc dans cette perspective intégrée que, dans les prochains paragraphes, nous aborderons la problématique spécifique de recherche nous préoccupant.

Selon les modèles retenus (Ashforth, 2001; Atchley, 1976; Beehr, 1986; Gall, Evans et Howard, 1997), le processus de la retraite comporte deux principales phases, celles de la préretraite et de la retraite, durant lesquelles l'individu doit d'abord se défaire d'un rôle pour lequel il est reconnu pour ensuite en apprivoiser un nouveau. Bien que des facteurs tels que l'âge, la santé, les ressources financières et la diversité des centres d'intérêts favorisent la concrétisation du projet de retraite (Adams, 1999; Hansson et al., 1997), selon Atchley (1976), c'est durant la phase de préretraite que le protagoniste peut avoir une attitude négative. Dans les faits, il s'agit d'une période d'insécurité durant laquelle celui s'y préparant physiquement et psychologiquement démontre moins de résistance, donc une attitude plus positive à l'égard de son éventuelle retraite (Lindbo et Schultz, 1998; Mutran, Reitzes et Fernandez, 1997). Dans une perspective de transition de rôle, la période de préretraite en est une de décristallisation fort complexe (Ashforth, 2001). Par exemple, selon Ebaugh (1988), celle-ci serait caractérisée par une continuelle évaluation du rôle existant, par une constante recherche de l'autorisation d'autrui de même que par l'identification d'un nouveau rôle dans lequel l'individu cherche à se reconnaître.

Revue internationale P.M.E., vol. 18, nºs 3-4, 2005

(C) 2005 - Presses de l'Université du Québec

Édifice Le Delta I, 2875, boul. Laurier, bureau 450, Sainte-Foy, Québec G1V 2M2 • Tél.: (418) 657-4399 - www.puq.ca

Tiré de: Revue internationale P.M.E., vol. 18, nos $3-4$, sous la direction de Louis Raymond • PME1803N

Tous droits de reproduction, de traduction et d'adaptation réservés 
Quant à la seconde phase, celle-ci se distingue par le passage effectif à la retraite. Durant les premiers mois, pouvant enfin vaquer à toutes les activités négligées durant le temps où il était au travail, le nouveau retraité vit une période euphorique (Atchley, 1976). Mais, pour plusieurs, retomber sur terre ne serait qu'une question de temps. Selon Atchley (1976), plus le nouveau retraité s'investissait dans sa vie professionnelle, plus il est susceptible de désenchanter rapidement. Dans une perspective de transition de rôle, cela fait partie du cours normal des choses. Selon Ashforth (2001), dès le moment où le nouveau retraité prend contact avec son nouveau rôle, il vit un état de choc, dont le plus significatif provient d'une prise de conscience de la teneur illusoire du portrait qu'il s'en était fait avant même de l'assumer. Ne pouvant s'identifier ni à son ancien, ni à son nouveau rôle, celui-ci se trouve alors dans un état d'esprit ambigu provoqué par l'absence de repères nécessaires à son équilibre. Pour finir, selon la littérature consultée, c'est après cette période de déséquilibre que le retraité se prend en charge et adopte de nouvelles routines, de nouvelles habitudes de vie dans lesquelles, y ayant trouvé un sens, il se sent confortable et reconnu à juste titre par son entourage immédiat (Ashforth, 2001; Atchley, 1976).

À la lumière de ce que nous venons d'exposer, il nous est maintenant possible de comprendre que le processus de désengagement du prédécesseur puisse s'échelonner sur deux périodes plutôt que sur une seule, soit celles du règne conjoint et du désengagement pendant lesquelles il procéderait à une profonde refonte de ses rôles au fur et à mesure que son successeur prend sa place. Mais comment le fait-il? Comment vit-il la période durant laquelle il doit partager sa place avec son successeur? Comment se sent-il après s'être retiré de son poste de PDG? Vit-il cette période d'euphorie dont on parle dans la littérature? Vit-il un choc à l'entrée? Comment réorganise-t-il sa vie après avoir laissé la gouvernance de l'entreprise entre les mains de sa progéniture? Voilà quelques questions auxquelles nous tenterons de répondre après avoir présenté la méthodologie de la recherche que nous avons privilégiée dans le cadre de cette étude.

\section{La méthodologie de la recherche}

Comme nous avions pour principal objectif de comprendre un phénomène ne pouvant exister que dans le contexte dans lequel il évolue, parmi les stratégies de recherche utilisées dans les sciences de la gestion, celle d'étude de cas semblait la plus appropriée (Yin, 1994). Pour nous, comprendre comment le prédécesseur vit cette période déterminante ne pouvait se faire qu'en optant pour une compréhension en profondeur d'un nombre de cas limité auxquels, en revanche, nous pouvions porter une attention des plus particulières. C'est donc dans cette perspective que nous avons cherché des entreprises ayant vécu leur premier transfert générationnel depuis un minimum de deux ans et dans lesquelles au moins un membre de la famille détenait le contrôle de la propriété et de la gestion. Au reste, pour s'assurer

Revue internationale P.M.E., vol. 18, n ${ }^{\text {os }} 3-4,2005$

(C) 2005 - Presses de l'Université du Québec

Édifice Le Delta I, 2875, boul. Laurier, bureau 450, Sainte-Foy, Québec G1V 2M2 - Tél.: (418) 657-4399 - www.puq.ca

Tiré de: Revue internationale P.M.E., vol. 18 , nos $3-4$, sous la direction de Louis Raymond - PME1803N

Tous droits de reproduction, de traduction et d'adaptation réservés 
d'étudier des entreprises où, selon nous, la succession était réussie, les prédécesseurs devaient s'être retirés de leur propre chef, être en bonne santé physique et morale et, surtout, être satisfaits de leur nouvelle vie.

Au Québec, aucune banque de données sur les entreprises familiales n'étant disponible, c'est par l'entremise du réseau de contacts, du dépouillement des publications d'affaires et de la navigation sur Internet qu'ont d'abord été relevées 139 entreprises. Pour des raisons de manque de représentativité liées à leur taille (trop petite ou trop grande) ou à leur situation géographique trop éloignée, 61 entreprises ont rapidement été écartées. Des 78 entreprises retenues pour contact téléphonique, 54 ont ensuite été rejetées. Dix-neuf étaient encore gérées par les fondateurs; huit avaient été transférées pour des raisons autres que celles nous intéressant (entre autres, certaines avaient été transférées à la suite du décès du fondateur); 12 étaient passées à la deuxième génération depuis plus de 10 ans; 10 à la troisième génération et cinq à la quatrième. Des 24 entreprises admissibles, 19 ayant refusé de participer, seules cinq ont fait partie du projet de recherche. Décrites au tableau 1, au moment de nos rencontres, les entreprises retenues œuvraient dans différents secteurs d'activité, généraient un chiffre d'affaires annuel variant entre 1 à 20 millions de dollars canadiens et employaient entre 24 et 190 personnes. Enfin, en ce qui concerne le transfert de la propriété, la manière dont chacune se distingue est, là aussi, remarquable notamment en ce qui concerne la manière dont il a été partagé entre les successeurs.

Tableau 1

Les entreprises participantes au projet de recherche

\begin{tabular}{|c|c|c|c|c|c|}
\hline & $\begin{array}{c}\text { Entreprise } \\
\text { Auger }\end{array}$ & $\begin{array}{c}\text { Entreprise } \\
\text { Bergeron }\end{array}$ & $\begin{array}{c}\text { Entreprise } \\
\text { Forest }\end{array}$ & $\begin{array}{c}\text { Entreprise } \\
\text { Mercier }\end{array}$ & $\begin{array}{c}\text { Entreprise } \\
\text { Sauvé }\end{array}$ \\
\hline Secteur d'activité & Construction & Services & Manufacturier & Détail & Manufacturier \\
\hline Chiffre d'affaires annuel & 1 à 3 M\$ & 10 à 15 M\$ & 18 à 20 M\$ & 1 à $3 \mathrm{M} \$$ & 3 à 5 M\$ \\
\hline Nombre d'employés & 24 & 190 & 125 & 30 & 28 \\
\hline $\begin{array}{l}\text { Nombre de générations } \\
\text { dans l'entreprise }\end{array}$ & 3 & 2 & 3 & 2 & 2 \\
\hline Fondation & 1964 & 1952 & 1964 & 1965 & 1978 \\
\hline Phase 3: Règne conjoint & 1997 & 1989 & 1990 & 1990 & 1997 \\
\hline Phase 4: Désengagement & 2000 & 1995 & 1997 & 1998 & 2000 \\
\hline Successeur(s) & 2 fils & 3 filles & 2 fils/1 fille & 1 fille & 3 fils \\
\hline $\begin{array}{l}\text { Prédécesseur(s)/ } \\
\text { fondateur(s) }\end{array}$ & Père & Père & Père & Mère & Père \\
\hline $\begin{array}{l}\text { État du transfert de la } \\
\text { propriété au moment de } \\
\text { nos rencontres }\end{array}$ & $\begin{array}{l}\text { P: p. c. } \\
\text { S1: } 50 \% \\
\text { S2: } 50 \%\end{array}$ & $\begin{array}{l}\text { P: p. c. } \\
\text { S1: } 34 \% \\
\text { S2: } 33 \% \\
\text { S3: } 33 \%\end{array}$ & $\begin{array}{l}\text { P: }: \text {. c. } \\
\text { S1: } 54 \% \\
\text { S2: } 19 \% \\
\text { S3: } 27 \%\end{array}$ & $\begin{array}{l}\text { P: } 51 \% \\
\text { S1: } 49 \%\end{array}$ & $\begin{array}{l}\text { P: p. c. } \\
\text { S1: } 33,3 \% \\
\text { S2: } 33,3 \% \\
\text { S3: } 33,3 \%\end{array}$ \\
\hline \multicolumn{6}{|c|}{$\begin{array}{l}\text { P: Prédécesseur/p.c.: parts de contrôle. } \\
\text { S1: Successeur occupant le poste de PDG. } \\
\text { S2 ou S3: Autres successeurs occupant des postes à la direction. }\end{array}$} \\
\hline
\end{tabular}

Revue internationale P.M.E., vol. 18, $\mathrm{n}^{\text {os }} 3-4,2005$

(C) 2005 - Presses de l'Université du Québec

Édifice Le Delta I, 2875, boul. Laurier, bureau 450, Sainte-Foy, Québec G1V 2M2 - Tél.: (418) 657-4399 - www.puq.ca

Tiré de: Revue internationale P.M.E., vol. 18 , $\mathrm{n}^{\text {os }} 3-4$, sous la direction de Louis Raymond $\cdot$ PME1803N

Tous droits de reproduction, de traduction et d'adaptation réservés 
Pour recueillir les données, des rencontres individuelles d'une durée d'une heure et demie chacune ont eu lieu avec tous les successeurs, tous les prédécesseurs et un tiers pour chacune des cinq entreprises. Après avoir été informés des objectifs de la recherche, c'est avec l'autorisation des interviewés que les 22 entrevues ont intégralement été enregistrées sur minicassettes. Dans le respect de la démarche proposée lors d'entrevues semi-structurées, la chercheure a utilisé une maquette l'assurant de recueillir les informations sur les environnements internes et externes des entreprises, sur les transferts afférents à l'ensemble du processus de la succession, sur la phase du désengagement et sur la manière dont les prédécesseurs vivaient cette période de leur vie. Dès que possible, toutes les entrevues ont été retranscrites mot à mot par la chercheure elle-même lui permettant ainsi de faire une première analyse et, le cas échéant, d'ajuster le tir lors d'entrevues subséquentes. Enfin, selon une liste de codes prédéterminés, les données ont initialement été traitées à l'aide du logiciel Atlas.ti et retraitées au fur et à mesure que de nouveaux codes émergeaient. Afin de nous assurer d'une interprétation la plus fidèle possible des représentations des participants (Huberman et Miles, 1994), les résultats préliminaires ont été présentés à deux prédécesseurs et à trois successeurs provenant de trois des entreprises participantes. Ils ont émis leurs commentaires et nous avons ensuite rédigé les schémas et les rapports ayant permis l'élaboration des résultats présentés ci-après.

\section{Le processus de désengagement du prédécesseur: les résultats}

Bien que chacune des entreprises faisant partie de l'étude ait sa propre histoire, plusieurs points communs dans les parcours des prédécesseurs sont notables. D'abord, dans tous les cas, ce sont les prédécesseurs qui ont clairement proposé à leur progéniture de prendre la relève de l'entreprise. Sauf pour la prédécesseure de l'entreprise Mercier, pour qui la démarche résulte de l'acquisition du commerce adjacent, pour les prédécesseurs des entreprises Auger, Bergeron, Forest et Sauvé, voyant la soixantaine arriver à grands pas, c'était plus une question de timing. Se sentant prêts à passer à une nouvelle étape de leur vie, les prédécesseurs n'ont toutefois pas bougé tant et aussi longtemps qu'au moins un de leurs enfants n'a verbalisé son réel intérêt pour relever un tel défi. Pour certains, la réponse a été immédiate tandis que pour d'autres, il a fallu attendre plus de six mois. Mais, dès le moment où les successeurs ont signalé leur intérêt pour prendre la relève de l'entreprise familiale, que s'est-il passé?

\subsection{Le processus de désengagement du prédécesseur durant la phase du règne conjoint}

D'abord, comme l'indique la littérature consultée (Churchill et Hatten, 1987; Handler, 1990; Hugron, 1992; Longenecker et Schoen, 1978), le règne conjoint fut une période durant laquelle les prédécesseurs ont appris à laisser de la place à

Revue internationale P.M.E., vol. 18, n ${ }^{\text {os }} 3-4,2005$

(C) 2005 - Presses de l'Université du Québec

Édifice Le Delta I, 2875, boul. Laurier, bureau 450, Sainte-Foy, Québec G1V 2M2 - Tél.: (418) 657-4399 - www.puq.ca

Tiré de: Revue internationale P.M.E., vol. 18, nos $3-4$, sous la direction de Louis Raymond - PME1803N

Tous droits de reproduction, de traduction et d'adaptation réservés 
leurs successeurs, et ce, même s'ils demeuraient les premiers responsables de leur entreprise. Hormis déléguer de plus en plus de responsabilités, une des premières réactions des prédécesseurs a été de diminuer leur temps de présence sur les lieux d'affaires tout en s'assurant de garder un contrôle serré sur les activités quotidiennes de leur organisation. Pour eux, mettre en place des moyens leur permettant de se distancier graduellement de la gouvernance de leur entreprise reposait sur deux principales raisons. La première était de garder contact avec la réalité de leur entreprise, leur permettant ainsi d'intervenir rapidement lorsque la situation l'exigeait, tandis que la seconde consistait à évaluer les compétences managériales des futurs dirigeants. C'est en effet durant la phase du règne conjoint - ayant selon les cas, duré entre trois et huit ans - que les successeurs ont dû prouver aux prédécesseurs (Cabera-Suarez, De Saa-Perez et Garcia-Almeida, 2001; Fiegener et al., 1996) leur engagement envers l'organisation (Chrisman, Chua et Sharma, 1998), leur capacité à prendre la place leur revenant en développant à la fois leur crédibilité et leur légitimité tant auprès de leurs prédécesseurs que de leurs pairs (Barach et al., 1988; Cabera-Suarez, De Saa-Perez et Garcia-Almeida, 2001), de même que leur capacité à gérer efficacement l'entreprise pour laquelle ils étaient destinés à prendre les rênes (Sharma, Chrisman et Chua, 2003).

Autrement, ce qui distingue les prédécesseurs auxquels nous nous sommes intéressée résulte de leur implication dans les processus d'intégration et de préparation de leurs successeurs. En vérité, à partir du moment où ceux-ci ont intégré l'entreprise pour en assurer la relève, tous les prédécesseurs se sont sentis investis d'une mission. Selon eux, ils avaient une part de responsabilité dans la réussite du projet, particulièrement envers celui destiné à les remplacer dans leur poste. Bien sûr, du point de vue des prédécesseurs, il était aussi de la responsabilité des successeurs de se prendre en main et de faire leurs preuves. Mais la manière dont ils se sont impliqués auprès de leurs successeurs les a conduits à relever de nouveaux défis avec lesquels aucun n'était familier. Par exemple, c'est durant la période du règne conjoint que les prédécesseurs ont, en ce qui nous concerne, assumé des rôles de collaborateur, d'enseignant, de mobilisateur et de superviseur pour préparer leurs successeurs à prendre la direction de l'entreprise. Et c'est en intégrant leurs successeurs dans les environnements dans lesquels ils étaient appelés à évoluer que les prédécesseurs ont aussi endossé ceux de passeur et de répondant (Cadieux et Lorrain, 2004). Mais attention! Même si les cinq prédécesseurs ont fait preuve d'ouverture et de disponibilité envers leurs successeurs durant le temps où ils devaient faire un bout de chemin ensemble, se défaire des comportements associés au rôle de dirigeant d'entreprise auquel ils étaient habitués n'a pas été aussi simple. Selon les dires des successeurs, c'est durant cette période que les prédécesseurs sont le plus intervenus dans les décisions qu'ils avaient prises. Selon eux, cette attitude de la part des prédécesseurs a eu un impact considérable sur leur capacité à prendre leur place auprès des employés pour qui les directives provenant des prédécesseurs l'emportaient.

Revue internationale P.M.E., vol. 18, nºs 3-4, 2005

(C) 2005 - Presses de l'Université du Québec

Édifice Le Delta I, 2875, boul. Laurier, bureau 450, Sainte-Foy, Québec G1V 2M2 • Tél.: (418) 657-4399 - www.puq.ca

Tiré de: Revue internationale P.M.E., vol. 18, nos $3-4$, sous la direction de Louis Raymond • PME1803N

Tous droits de reproduction, de traduction et d'adaptation réservés 
Finalement, avant de passer à la phase du désengagement, certains prédécesseurs ont ressenti le besoin de se rassurer dans leurs démarches respectives. À ce chapitre, outre des rapports écrits leur permettant d'évaluer les compétences managériales et la courbe d'apprentissage de leurs successeurs, les prédécesseurs des entreprises Auger, Bergeron et Mercier ont partagé leurs préoccupations avec autrui. Inquiets, ils se sentaient confrontés à une nouvelle réalité pour laquelle ils cherchaient des repères pouvant provenir des expériences vécues par leurs pairs.

\subsection{Le processus de désengagement du prédécesseur durant la phase du désengagement}

En ce qui concerne les cinq prédécesseurs, tous comprenaient une chose. Assurer la pérennité de leur entreprise les obligeait à prendre des décisions et à poser des gestes leur permettant de cristalliser leur démarche débutée depuis que leurs successeurs avaient intégré l'entreprise pour en prendre la relève. Or, même si les cinq ont réussi à passer à l'acte, tous ne l'ont pas fait ni pour les mêmes raisons, ni dans les mêmes circonstances. Alors que les prédécesseurs des entreprises Auger, Forest et Sauvé ont amorcé la phase du désengagement au moment où ils se sont sentis prêts à le faire, ceux des entreprises Bergeron et Mercier l'ont fait parce qu'ils y ont été obligés. En revanche, dès que leur décision de se retirer de la gouvernance de leur entreprise a été définitive, les cinq prédécesseurs ont posé certains gestes leur permettant de sanctionner ce passage déterminant pour la pérennité de l'organisation. Par exemple, tandis que les prédécesseurs des entreprises Bergeron, Forest et Sauvé ont officiellement annoncé la nomination de leur successeur au poste de président-directeur général et, par la même occasion, leur propre mise à la retraite, tous l'ont aussi sanctionné en procédant au transfert de la propriété.

Dans les cas nous intéressant, même si les décisions prises et les gestes posés par les prédécesseurs ont permis d'amorcer la phase du désengagement, ces derniers étaient loin d'avoir atteint la tranquillité d'esprit à laquelle ils aspiraient. En effet, des deux dernières phases du processus de la succession, la dernière est probablement celle où les prédécesseurs ont le plus été ébranlés. D'abord, bien que se sentant prêts à passer à cette étape cruciale de leur vie, les cinq prédécesseurs disent avoir vécu une période durant laquelle ils se sont sentis désorientés et désorganisés. En réalité, dès le moment où ils se sont retirés de la gouvernance de leur entreprise, tous ont eu le sentiment d'être, d'une part, inutiles et, d'autre part, démunis devant leur incapacité à trouver les comportements à adopter dans une situation tout à fait nouvelle pour eux. Dans certains cas, il faudra plus de six mois pour que les prédécesseurs s'en remettent.

Amorcée depuis entre deux et sept ans selon les cas, la phase du désengagement comporte de nombreux changements. D'abord, dès le moment où les successeurs ont officiellement pris en charge la direction de l'entreprise, tous ont

Revue internationale P.M.E., vol. 18, n ${ }^{\text {os }} 3-4,2005$

(C) 2005 - Presses de l'Université du Québec

Édifice Le Delta I, 2875, boul. Laurier, bureau 450, Sainte-Foy, Québec G1V 2M2 - Tél.: (418) 657-4399 - www.puq.ca

Tiré de: Revue internationale P.M.E., vol. 18, nos $3-4$, sous la direction de Louis Raymond - PME1803N

Tous droits de reproduction, de traduction et d'adaptation réservés 
remanié la structure organisationnelle, professionnalisé les processus de gestion et adopté de nouvelles stratégies leur permettant de développer de nouveaux marchés. Or, les résultats positifs en découlant ont amené les prédécesseurs à définitivement reconnaître les compétences de leurs successeurs et, par voie de conséquence, leurs propres limites. Dans une perspective freudienne de deuil, cela fait partie intégrante du processus du désengagement psychologique individuel des prédécesseurs (Pailot, 2000). Heureux et fiers d'avoir réussi à assurer la pérennité de leur entreprise, tous ont fini par adopter une attitude plus positive envers leur nouvelle vie. Ce nouvel état d'esprit amène doucement les prédécesseurs à perdre contact avec certaines des nouvelles réalités de leur entreprise dont la destinée dépend des successeurs en poste.

Maintenant, depuis qu'ils se sont retirés de la gouvernance de leur entreprise, comment les cinq prédécesseurs auxquels nous nous sommes intéressés ont-ils réorganisé leur vie? D' abord, comme l'indique la littérature consultée (Aronoff et Ward, 1992; Christensen, 1979; Hugron, 1992; Hunt et Handler, 1999), nos résultats montrent que les prédécesseurs gardent contact avec l'entreprise, et ce, même s'ils ne sont plus à la barre. Premièrement, qu'ils soient demeurés président ou nommés président du conseil d'administration, tous ont gardé un droit de veto leur permettant d'intervenir dans les décisions pouvant porter préjudice à l'entreprise. C'est d'ailleurs à ce titre qu'ils reçoivent les états financiers et d'autres rapports leur permettant de suivre l'état de santé financière de l'entreprise et de poser des questions lorsqu'ils en ressentent le besoin. Deuxièmement, de façon ponctuelle, les cinq prédécesseurs se rendent toujours sur les lieux d'affaires de l'entreprise. Ils profitent d'ailleurs du temps où ils sont dans l'entreprise pour observer, s'informer et s'assurer que les valeurs auxquelles ils tiennent sont perpétuées.

Même si la phase du désengagement est caractérisée par une reconnaissance unilatérale d'une nouvelle direction, nos résultats montrent que les prédécesseurs y ont toujours une place privilégiée. Par exemple, lorsque nous les avons interrogés, les successeurs et les tiers revenaient souvent sur le rôle qu'avait joué le prédécesseur dans l'histoire de l'entreprise. Pour eux, le prédécesseur est un héros. C'est lui qui a bâti l'entreprise. C'est lui qui a trimé dur. Et c'est lui qui a surmonté les difficultés s'étant présentées en cours de route. Sans eux, l'entreprise à laquelle nous nous intéressions n'existerait même pas. Quatre prédécesseurs continuent d'ailleurs à interagir avec certains acteurs de l'environnement externe avec qui ils ont développé des liens de confiance sans que cela ne nuise d'aucune manière au bon déroulement de l'organisation. Qui plus est, sous prétexte qu'ils ont encore beaucoup d'influence auprès de certains clients ou fournisseurs, on leur demande d'intervenir dans les cas problématiques.

De plus, nos résultats indiquent qu'il pourrait exister deux parcours dans la manière dont les prédécesseurs réorganisent leur vie. Dans le premier, respectés

Revue internationale P.M.E., vol. 18, ${ }^{\text {os }} 3-4,2005$

(C) 2005 - Presses de l'Université du Québec

Édifice Le Delta I, 2875, boul. Laurier, bureau 450, Sainte-Foy, Québec G1V 2M2 • Tél.: (418) 657-4399 - www.puq.ca

Tiré de: Revue internationale P.M.E., vol. $18, \mathrm{n}^{\text {os }} 3-4$, sous la direction de Louis Raymond • PME1803N

Tous droits de reproduction, de traduction et d'adaptation réservés 
pour leurs compétences techniques, c'est en s'impliquant physiquement à la production ou à la recherche et au développement que les prédécesseurs des entreprises Auger, Forest et Mercier agissent, en ce qui nous concerne, comme support technique. N'ayant jamais été captivés par les tâches administratives, agir à ce titre leur permet de vaquer aux occupations qu'ils affectionnent. Quant au second parcours, se distinguant par leurs intérêts pour la gestion, ceux des entreprises Bergeron et Sauvé agissent plutôt comme consultant. Leur rôle prend alors une forme pouvant correspondre à celle réservée à tout consultant ayant les compétences pour mener à terme le projet pour lequel il est mandaté. Enfin, bien que les prédécesseurs aient délibérément abandonné les activités pour lesquelles ils n'avaient jamais eu beaucoup d'intérêt, tous n'ont pas non plus redistribué leur temps de la même manière. Alors que les prédécesseurs des entreprises Bergeron, Mercier et Sauvé consacrent la majorité de leur temps à des activités de loisirs qu'ils affectionnent depuis plusieurs années, ceux des entreprises Auger et Forest l'ont surtout redistribué autour d'activités organisationnelles de nature plus opérationnelle les ayant toujours passionnés.

Enfin, durant la phase du désengagement, nous avons aussi constaté que les prédécesseurs assument de nouveaux rôles auprès de leurs successeurs. Par exemple, préoccupés de préserver l'harmonie entre tous les membres de la famille, les prédécesseurs des entreprises Auger, Bergeron, Forest et Sauvé agissent comme arbitres, conciliateurs, modérateurs ou médiateurs dans des situations pouvant porter préjudice à l'entreprise et à la famille. Du reste, ayant commencé à prendre forme durant la phase du règne conjoint, la relation privilégiée entre les prédécesseurs et celui ou celle parmi leurs enfants faisant maintenant office de p.-d.g. est, durant la phase du désengagement, solidement installée. Les nouveaux dirigeants en poste continuent de tirer profit d'une des principales ressources à leur portée leur permettant d'évacuer leurs préoccupations qui, dans un passé récent, ont été celles de leurs prédécesseurs maintenant devenus leurs confidents.

\subsection{Le processus de désengagement du prédécesseur: proposition d'un modèle de réussite}

À la lumière des résultats présentés, nous pouvons comprendre dans quelle mesure, pour le prédécesseur, se désengager de l'entreprise pour laquelle il a été responsable pendant bon nombre d'années peut effectivement correspondre à un rituel de transition de rôle durant lequel la période de sortie de rôle peut être caractérisée par certains rites de séparation tandis que celle de l'entrée de rôle peut l'être par d'autres rites d'incorporation (Ashforth, 2001; Atchley, 1976). En effet, comme l'illustre la figure 1 , pour les cas à l'étude, la phase du règne conjoint amorcée après la propre initiative des prédécesseurs a amené ceux-ci à se distancier de leur ancien rôle et à en assumer de nouveaux auprès de leurs successeurs, d'une part,

Revue internationale P.M.E., vol. 18, $\mathrm{n}^{\text {os }} 3-4,2005$

(C) 2005 - Presses de l'Université du Québec

Édifice Le Delta I, 2875, boul. Laurier, bureau 450, Sainte-Foy, Québec G1V 2M2 - Tél.: (418) 657-4399 - www.puq.ca

Tiré de: Revue internationale P.M.E., vol. 18, nos 3-4, sous la direction de Louis Raymond • PME1803N

Tous droits de reproduction, de traduction et d'adaptation réservés 
pour les préparer à prendre les rênes de l'entreprise et, d'autre part, pour les intégrer à leurs nouveaux environnements. Devant, dès lors, s'habituer à prendre de moins en moins de place et à partager pouvoir et autorité, c'est durant cette période cruciale que sont par ailleurs apparues des résistances individuelles significatives lesquelles, néanmoins, se sont estompées au fur et à mesure que leurs successeurs ont fait leurs preuves.

Dans les cinq cas, il a fallu une deuxième série d'événements déclencheurs pour amener les prédécesseurs à poser des gestes concrets permettant le passage officiel à la dernière phase du processus de la succession, celle du désengagement. Bien que se disant prêts, ils ont tout de même été amenés à vivre une période durant laquelle ils se sont sentis désorganisés et inutiles. Ensuite, est venu la période où tous ont été en mesure de reconnaître ouvertement à la fois les compétences de leurs successeurs ainsi que leurs propres limites. Ayant réussi à recentrer leurs énergies autour des activités les ayant toujours passionnés, tous se sont par ailleurs appropriés de nouveaux rôles. À ce chapitre, deux types de rôles peuvent leur être attribués. D'une part, il y a ceux qu'ils endossent auprès de l'organisation à titre d'administrateur, de symbole, d'observateur, de protecteur, d'intermédiaire, de support technique et/ou de consultant et, d'autre part, il y a ceux qu'ils jouent auprès de leurs successeurs, que ce soit à titre de facilitateur ou de confident (Cadieux et Lorrain, 2004).

Figure 2

\section{Le processus de désengagement des prédécesseurs: un modèle de réussite}

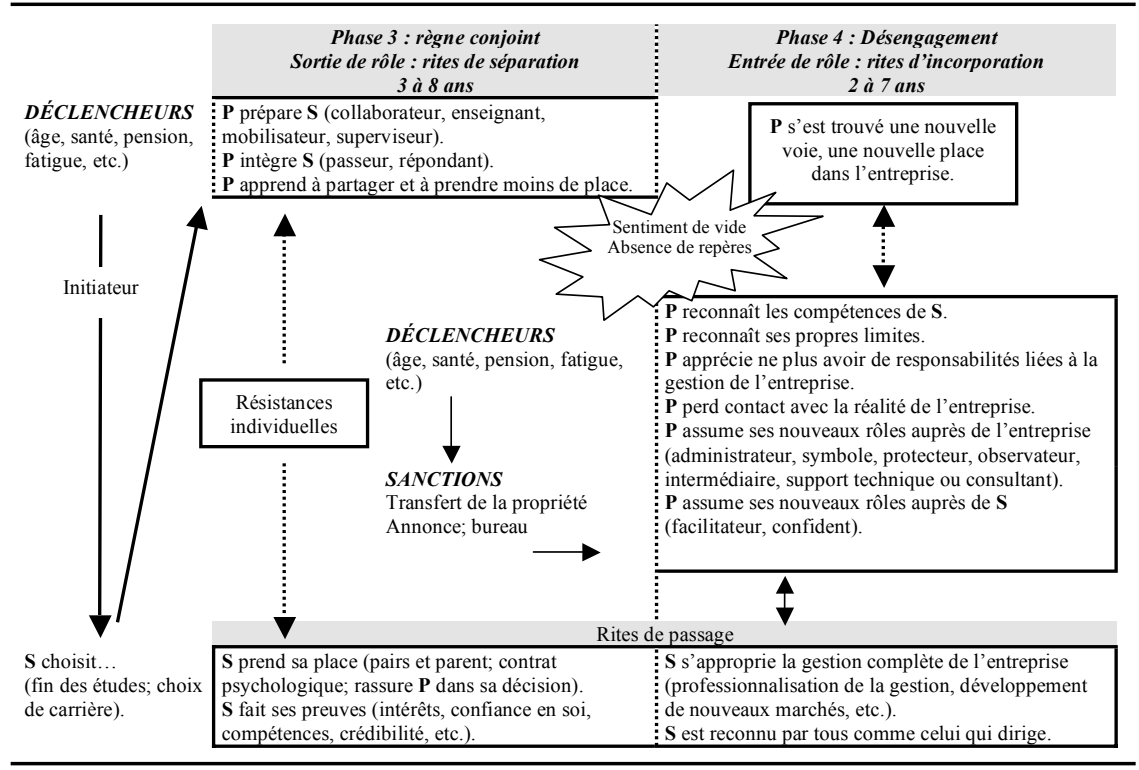

Revue internationale P.M.E., vol. 18, nºs 3-4, 2005

(C) 2005 - Presses de l'Université du Québec

Édifice Le Delta I, 2875, boul. Laurier, bureau 450, Sainte-Foy, Québec G1V 2M2 - Tél.: (418) 657-4399 - www.puq.ca

Tiré de: Revue internationale P.M.E., vol. 18, nos $3-4$, sous la direction de Louis Raymond - PME1803N

Tous droits de reproduction, de traduction et d'adaptation réservés 


\section{Discussion et conclusion}

D'entrée de jeu, les résultats présentés concordent avec ceux déjà obtenus dans le cadre d'études empiriques menées auprès de différentes populations de hauts dirigeants d'entreprises qu'elles soient familiales ou non (Christensen, 1979; Handler, 1990; Levinson et Wofford, 2000; Longenecker et Schoen, 1978; Pailot, 2000; Santora et Sarros, 1995; Sonnenfeld, 1988). Le lien est direct. Quelle que soit la nature ou la taille de l'entreprise, parce que cela implique plusieurs changements substantiels des rôles et des activités leur ayant prodigué un statut particulier pendant de si nombreuses années, pour les prédécesseurs, se désengager de la gouvernance de leur entreprise prend du temps, est difficile et douloureux.

Même si les prédécesseurs sous étude ont réussi à se désengager de la gouvernance de leur entreprise, selon les successeurs, cela ne s'est pas fait sans heurts. Comme nous l'avons souligné, la majorité disent en effet avoir vécu une période critique durant laquelle le prédécesseur a montré des difficultés à déléguer et à lâcher prise sur bon nombre de décisions. Or, lorsque nous en avons discuté avec les prédécesseurs, ceux-ci en ont parlé bien différemment. Pour eux, une chose était claire. Avant qu'ils ne laissent leurs successeurs complètement libres, ils devaient avoir fait leurs preuves! En ce qui nous concerne, ce constat procure un éclaircissement sur les sources de résistance pouvant provenir du prédécesseur et de la qualité de la relation existant entre ce dernier et le successeur (Handler et Kram, 1988). Par exemple, une des difficultés soulevées par Handler (1989) proviendrait des périodes durant lesquelles le successeur n'évolue pas au même rythme que son prédécesseur dans leurs processus de transitions de rôles respectifs. Or, à la lumière des résultats obtenus dans notre recherche, il est possible de supposer que cette impression de manque de synchronisme puisse s'estomper au fur et à mesure que chacun prend conscience de la différence pouvant exister entre le rythme du processus d'apprentissage du successeur et celui du désengagement du prédécesseur. De plus, contrairement à ce que stipulent certains auteurs consultés (Christensen, 1979; Kets de Vries, 1993; Sonnenfeld, 1988), ce ne serait pas le passage à la phase du désengagement qui serait le plus susceptible de provoquer de la résistance chez le prédécesseur, mais bien, comme le soulèvent d'autres (Davis et Harveston, 1998; Lansberg et Astrachan, 1994; Morris et al., 1997; Murray, 2003), l'absence de l'établissement d'une relation empreinte de complicité, de confiance et de respect entre le prédécesseur et le successeur appelés à travailler en étroite collaboration dès le début de la phase du règne conjoint.

Des deux phases du modèle de réussite du processus de désengagement proposé, il semble que la seconde ait été la plus bouleversante pour les prédécesseurs. Oui, ils ont fait preuve de résistance durant la mise en place de leurs successeurs. Et oui, ils étaient prêts à passer à l'étape suivante. Mais, à leur grande surprise, la partie n'était pas encore gagnée. Par exemple, même si tous se disaient

Revue internationale P.M.E., vol. 18, ${ }^{\text {os }} 3-4,2005$

(C) 2005 - Presses de l'Université du Québec

Édifice Le Delta I, 2875, boul. Laurier, bureau 450, Sainte-Foy, Québec G1V 2M2 - Tél.: (418) 657-4399 - www.puq.ca

Tiré de: Revue internationale P.M.E., vol. 18 , nos $3-4$, sous la direction de Louis Raymond • PME1803N

Tous droits de reproduction, de traduction et d'adaptation réservés 
heureux dans leur nouvelle vie, lorsqu'ils ont parlé de la période où ils ont cédé leur entreprise à leur progéniture, c'est avec beaucoup d'émotion qu'ils l'ont fait. Pour eux, indépendamment de la fierté qu'ils en retiraient, passer à l'acte officialisait du même coup leur mise à la retraite d'une entreprise envers laquelle ils ressentaient toujours un profond sentiment d'attachement et un fort degré d'identification, et cela, indépendamment du nombre d'années écoulées depuis l'amorce de la phase du désengagement. Ainsi, à la lumière des résultats présentés précédemment, sommes-nous en mesure de comprendre que même dans les cas où les prédécesseurs réussissent à se désengager de la gouvernance de leur entreprise, ceux-ci vivront une période difficile durant laquelle ils doivent faire le deuil de leur rôle, de leur entreprise et de leur statut, même s'ils s'y étaient préparés.

La présente recherche comporte des limites dont la principale découle fondamentalement de la stratégie de recherche privilégiée. En effet, même si certains des résultats éclairent mieux le processus de désengagement du prédécesseur, le petit nombre auquel nous nous sommes intéressés ne nous permet aucunement de généraliser les résultats obtenus, ce qui, en réalité, n'était pas non plus notre objectif. Ayant aussi volontairement choisi de limiter notre étude aux cas de PME familiales ayant réussi leur premier transfert générationnel, faute d'avoir répliqué les théories émergentes par le truchement d'au moins un cas contraire ou d'un cas atypique (Yin, 1994), nous devons admettre ne pas encore être en mesure ni de valider les énoncés émis, ni de comprendre ceux ayant échoué ou ceux ayant effectué les passages générationnels subséquents.

Parmi les auteurs consultés traitant de la succession dans les entreprises familiales, tous s'entendent sur un point. Le processus de la succession est de nature profondément interactive (Barnes et Hershon, 1976; Churchill et Hatten, 1987; Gersick et al., 1997; Handler, 1990; Holland et Boulton, 1984; Holland et Oliver, 1992; Hugron, 1992; Longenecker et Schoen, 1978). À cet égard, parmi les résultats présentés, nous avons compris combien les prédécesseurs réussissent à se désengager en très grande partie parce qu'ils ont l'assurance que leurs successeurs ont les compétences requises pour les remplacer. Pourtant, en considérant que pour assurer le passage générationnel les processus d'intégration et de préparation des successeurs sont aussi importants que celui de désengagement des prédécesseurs, ayant volontairement canalisé notre analyse sur un seul groupe d'acteurs, nous devons admettre ne pas avoir les réponses à plusieurs questions nécessaires à une meilleure compréhension du processus de la succession dans son ensemble. Par exemple, comprenant que pour assurer la relève, le successeur doive faire sa place tant auprès de son prédécesseur que de ses pairs, comment le fait-il? Comment réussit-il à se faire reconnaître à titre de dirigeant de l'entreprise? Au reste, comprenant qu'il soit important pour le prédécesseur de voir son successeur maîtriser tant les environnements interne qu'externe de l'organisation, comment ce dernier réussit-il à acquérir le bagage de connaissances requises pour lui permettre de développer de telles compétences?

Revue internationale P.M.E., vol. 18, $\mathrm{n}^{\text {ss }} 3-4,2005$

(C) 2005 - Presses de l'Université du Québec

Édifice Le Delta I, 2875, boul. Laurier, bureau 450, Sainte-Foy, Québec G1V 2M2 - Tél.: (418) 657-4399 - www.puq.ca

Tiré de: Revue internationale P.M.E., vol. 18, nos $3-4$, sous la direction de Louis Raymond • PME1803N

Tous droits de reproduction, de traduction et d'adaptation réservés 
Lors de la révision de la littérature sur la retraite, certains des auteurs consultés stipulent qu'il s'agit d'un processus impliquant les autres membres de la famille, dont en particulier le conjoint (Henkens, 1999; Smith et Moen, 1998). À ce chapitre, les résultats d'une étude faite par Poza et Messer (2001) auprès de conjoints de dirigeants d'entreprises familiales révèlent comment ceux-ci peuvent effectivement endosser un rôle de médiateur auprès des membres de la famille durant le processus du transfert générationnel. Or, dans notre recherche, les conjoints des prédécesseurs participants ont volontairement été exclus de l'analyse, et ce, même si, selon quelques-uns des successeurs rencontrés, certains auraient effectivement eu une influence sur le déroulement du processus de la succession. Voilà une deuxième limite de taille ne nous permettant pas de répondre à des questions pourtant importantes. En effet, si les conjoints ont un ou plusieurs rôles à jouer durant le processus de la succession, en quoi consistent-ils? Comment ont-ils une influence sur la qualité de la relation qui s'établit entre le prédécesseur et le successeur? De plus, le fait que plusieurs conjoints s'impliquent dans l'organisation souvent dès le début des opérations (Danes et Olson, 2003; Poza et Messer, 2001) soulèvent d'autres questionnements encore. Par exemple, après avoir développé un sentiment d'appartenance et un degré d'identification à l'entreprise, comment les conjoints réussissent-ils, eux aussi, à faire le deuil du statut que cela leur procurait? Dans quelle mesure cela peut-il être source de conflits? de résistance? Enfin, comment cela peut-il avoir une influence sur le processus de désengagement des prédécesseurs?

Les modèles de réussite du transfert générationnel provenant d'une population de PME familiales ayant émergé après la Seconde Guerre mondiale sont rares. Toutefois, parce que les cinq prédécesseurs auxquels nous nous sommes intéressés ont réussi ce passage délicat, comprendre les difficultés qu'ils ont rencontrées et su surmonter durant les deux dernières phases du processus de la succession peut définitivement servir de repères pour ceux et celles appelés à vivre cette période sous peu, qu'il s'agisse des prédécesseurs, des successeurs, des membres de la famille ou des intervenants. Enfin, comprendre qu'il soit tout à fait possible pour les prédécesseurs d'accompagner leurs successeurs durant leur formation et comprendre qu'ils puissent encore être utiles après s'être officiellement retirés de la gouvernance de leur entreprise est, en ce qui nous concerne, inviter un des groupes d'acteurs les plus influents dans le contexte à relever de nouveaux défis auxquels ils ne sauront certainement pas résister.

\section{Bibliographie}

ADAMs, G.A. (1999), «Career-related variables and planned retirement age: an extension of Beehr's model», Journal of Vocational Behavior, vol. 55, nº 2, p. 221-235.

Allouche, J. et B. Amann (1998), «La confiance: une explication des performances des entreprises familiales», Économie et société, vol. 8, n 9, p. 129-154.

Revue internationale P.M.E., vol. 18, $\mathrm{n}^{\text {os }} 3-4,2005$

(C) 2005 - Presses de l'Université du Québec

Édifice Le Delta I, 2875, boul. Laurier, bureau 450, Sainte-Foy, Québec G1V 2M2 - Tél.: (418) 657-4399 - www.puq.ca

Tiré de: Revue internationale P.M.E., vol. 18 , nos $3-4$, sous la direction de Louis Raymond - PME1803N

Tous droits de reproduction, de traduction et d'adaptation réservés 
AronofF, C.E. et J.L. W ARD (1992), «Family business succession: the final test of greatness», Family Business Leadership Series, $\mathrm{n}^{\circ} 1$, Marietta, Georgia, Business Owners Resources.

Ashrorth, B.E. (2001), Role Transitions in Organizational Life: An Identity-Based Perspective, Mahwah, N.J., Lawrence Erlbaum Associates.

Atchley, R.C. (1976), The Sociology of Retirement, Cambridge, Shenkman Publishing.

Barach, J.A., J. Ganitsky, J.A. Carson et B.A. Doochin (1988), «Entry of the next generation: strategic challenge for family business», Journal of Small Business Management, vol. 26, $\mathrm{n}^{\circ}$ 2, p. 49-56.

Barnes, L.B. et S.A. Hershon (1976), «Transferring power in family business», Harvard Business Review, p. 105-114.

BEEHR, T.A. (1986), «The process of retirement: a review and recommandations for future investigations», Personnel Psychology, vol. 39, n 1, p. 31-55.

Cabera-Suarez, K., P. De SaA-Perez et D. Garcia-Almeida (2001), «The succession process form a resource and knowledge based view of the family firm», Family Business Review, vol. XIV, $\mathrm{n}^{\circ}$ 1, p. 37-47.

CAdieux, L. et J. Lorrain (2004), «La succession dans les PME familiales: vers une typologie de rôles endossés par le prédécesseur durant et après la mise en place de son successeur», Actes du Congrès international francophone sur la PME (CIFPME), Montpellier, octobre.

Chrisman, J.J., J.H. Chua et P. Sharma (1998), «Important attributes of successors in family businesses: an exploratory study», Family Business Review, vol. XI, n ${ }^{\circ}$, p. 19-34.

Christensen, C.R. (1979), Management Succession in Small and Growing Enterprises,

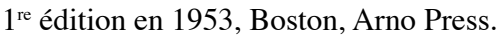

Churchill, N.C. et K.J. Hatten (1987), «Non-market based transfers of wealth and power: a research framework for family businesses », American Journal of Small Business, vol. 11, no 3, p. 51-64.

Cromie, S., B. Dunn, A. Sproull et D. Chalmers (2001), «Small firms with a family focus in the Scottish highlands and islands», The Irish Journal of Management, vol. 22, $\mathrm{n}^{\circ} 2$, p. 45-66.

Danes, S.M. et P.D. Olson (2003), «Women's role involvement in family businesses, business tensions, and business success », Family Business Review, vol. XVI, n 1 , p. 53-68.

Davis, P.S. et P.D. Harveston (1998), «The influence of family on the family business succession process : a multi-generational perspective», Entrepreneurship Theory and Practice, vol, 22, $\mathrm{n}^{\circ}$ 3, p. 31-53.

Ebaugh, H.R. (1988), Becoming an EX: The Process of Role Exit, Chicago, The University of Chicago Press.

FFI - Family Firm Institute (2003), Facts and Perspectives on Family Business, Family Firm Institute, saisi le 17 octobre de <www.ffi.org/looking/fbfacts_us.html>.

Revue internationale P.M.E., vol. 18, $\mathrm{n}^{\text {os }} 3-4,2005$

(C) 2005 - Presses de l'Université du Québec

Édifice Le Delta I, 2875, boul. Laurier, bureau 450, Sainte-Foy, Québec G1V 2M2 • Tél.: (418) 657-4399 - www.puq.ca

Tiré de: Revue internationale P.M.E., vol. 18, nos $3-4$, sous la direction de Louis Raymond - PME1803N

Tous droits de reproduction, de traduction et d'adaptation réservés 
Fiegener, M.K., B.M. Brown, R.A. Prince et K.M. File (1996), «Passing on strategic vision», Journal of Small Business Management, vol. 34, $\mathrm{n}^{\circ}$ 3, p. 15-26.

FortezA, J.A. et J.M. Prieto (1994), «Aging and work behaviour», dans H.-C. Triandis, M.D. Dunnette et L.M. Hough (dir.), Handbook of Industrial \& Organizational Psychology, volume 4, $2^{\mathrm{e}}$ édition, Palo Alto, Californie, Consulting Psychologists Press, p. 447-483.

GALL, T.L., D.R. Evans et J. HowARD (1997), «The retirement adjustment process: changes in the well-being of males retirees across time», Journal of Gerontology, Psychological Sciences, vol. 52B, no 3, p. 110-117.

Garcia-Alvarez, E. et J. LopeZ-Sintas (2001), «A taxonomy of founders based on values: the root of family business heterogeneity», Family Business Review, vol. XIV, n 3 , p. 209-230.

Gersick, K.E., J.A. Davis, M. McCollom Hampton et I. Lansberg (1997), Generation to Generation: Life Cycles of the Family Business, Boston, Harvard Business School Press.

Handler, W.C. (1989), Managing the Family Firm Succession Process: The Next-Generation Family Member's Experience, Thèse de doctorat, Université de Boston, UMI Dissertation Services.

HANDLER, W.C. (1990), «Succession in family firms: a mutual role adjustment between entrepreneur and next-generation family members», Entrepreneurship Theory and Practice, vol. 15, $\mathrm{n}^{\circ}$ 1, p. 37-51.

HANDLER, W.C. (1994), «Succession in family business: a review of the research», Family Business Review, vol. VII, n ${ }^{\circ}$ 2, p. 133-157.

HANDLER, W.C. et K.E. Kram (1988), «Succession in family firms: the problem of resistance», Family Business Review, vol. 1, nº 4, p. 361-381.

Hansson, R.O., P.D. DeKoekкoeK, W.M. Neece et D.W. Patterson (1997), «Successful aging at work: annual review 1992-1996. The older worker and transitions to retirement», Journal of Vocational Behavior, vol. 51, n 2, p. 202-233.

Henkens, K. (1999), «Retirement intentions and spousal support: a multi-actor approach», Journal of Gerontology, Social Sciences, vol. 54B, nº 2, p. S63-S73.

Holland, P.G. et W.R. Boulton (1984), «Balancing the family and the business in family business », Business Horizons, vol. 27, n 2, p. 16-21.

Holland, P.G. et J.E. Oliver (1992), «An empirical examination of the stages of development of family business», Journal of Business and Entrepreneurship, vol. 4, $\mathrm{n}^{\circ} 3$, p. 27-38.

Huberman, A.M. et M.B. Miles (1994), «Data management and analysis methods», dans Handbook of Qualitative Research, Thousand Oaks, Cal., Sage Publications, p. $428-444$.

Hugron, P. (1992), L'entreprise familiale: modèle de réussite du processus successoral, monographie publiée par l'Institut de recherches politiques et les Presses HEC.

Hunt, J.M. et W.C. Handler (1999), «The practices of effective family firm leaders», Journal of Developmental Entrepreneurship, vol. 4, n 2, p. 135-151.

Revue internationale P.M.E., vol. 18, $\mathrm{n}^{\text {os }} 3-4,2005$

(C) 2005 - Presses de l'Université du Québec

Édifice Le Delta I, 2875, boul. Laurier, bureau 450, Sainte-Foy, Québec G1V 2M2 - Tél.: (418) 657-4399 - www.puq.ca

Tiré de: Revue internationale P.M.E., vol. 18 , nos $3-4$, sous la direction de Louis Raymond $\cdot$ PME1803N

Tous droits de reproduction, de traduction et d'adaptation réservés 
JuLIEN, P.-A. (2000), L'entrepreneuriat au Québec: pour une révolution tranquille entrepreneuriale 1980-2000, Québec, Les éditions de la Fondation de l'entrepreneurship, $400 \mathrm{p}$.

KETS DE VRIES, M.F. (1993), «The dynamics of family controlled firms: the good and the bad news», Organizational Dynamics, vol. 21, p. 59-71.

LANSBERG, I. et J.H. Astrachan (1994), «Influence of family relationships on succession planning and training: the importance of mediating factors», Family Business Review, vol. VII, $n^{\circ} 1$, p. 39-59.

LEVInSON, H. et J.C. WOFFORD (2000), «Approaching retirement as the flexibility phase», The Academy of Management Executive, vol. 14, n 2, p. 84-95.

LindBo, T.L. et K.S. Schultz (1998), «The role of organizational culture and mentoring in mature worker socialization toward retirement», Public Productivity and Management Review, vol. 22, n 1, p. 49-59.

LONGENECKER, J.G. et J.E. SCHOEN (1978), «Management succession in the family business », dans C. Aronoff, J. Astrachan et J. Ward (1996), Family Business Sourcebook II, Georgia, Omnigraphics, p. 87-92.

Matthews, C.H., T.W. Moore et A.S. Fialko (1999), «Succession in the family firm: a cognitive categorization perspective», Family Business Review, vol. XII, n 2, p. 159-169.

Smith, D.B. et P. Moen (1998), «Spousal influence on retirement: his, her and their perception», Journal of Marriage and the Family, vol. 60, n 3, p. 734-744.

Morris, M.H., R.O.Williams, J.A. Allen et R.A. Avila (1997), «Correlates of success in family business in transition», Journal of Business Venturing, vol. 12, $\mathrm{n}^{\circ} 5$, p. $385-401$.

Murray, B. (2003), «The succession transition process: a longitudinal perspective», Family Business Review, vol. XVI, nº 1, p. 17-33.

Mutran, E.J., D.C. Reitzes et M.E. Fernandez (1997), «Factors that influence attitudes toward retirement», Research on Aging, vol. 19, n 3, p. 251-173.

PAILOT, P. (2000), «De la difficulté de l'entrepreneur à quitter son entreprise», dans T. Verstraete (dir.), Histoire d'entreprendre: Les réalités de l'entrepreneuriat, Colombelles, Éditions EMS Management et Société, p. 275-286.

PEAY, R.T. et G. DYER (1989), «Power orientations of entrepreneurs and succession planning», Journal of Small Business Management, vol. 27, n 1, p. 47-52.

Peiser, R.B. et L.M. Wooten, (1983), «Life-cycle changes in small family business», Business Horizons, p. 58-65.

Poza, E. et T. Messer (2001), «Spousal leadership and continuity in the family firm», Family Business Review, vol. XIV, $\mathrm{n}^{\circ}$ 1, p. 25-36.

SANTORA, J.C. et J.C. SARros (1995), «Mortality and leadership succession: a case study», Leadership \& Organization Development Journal, vol. 16, nº 7, p. 29-32.

Sharma, P., J. Chrisman et J. Chua (2003), «Succession planning as planned behaviour: some empirical results», Family Business Review, vol. XVI, n 1, p. 1-15.

Revue internationale P.M.E., vol. 18, $\mathrm{n}^{\mathrm{os}} 3-4,2005$

(C) 2005 - Presses de l'Université du Québec

Édifice Le Delta I, 2875, boul. Laurier, bureau 450, Sainte-Foy, Québec G1V 2M2 • Tél.: (418) 657-4399 - www.puq.ca

Tiré de: Revue internationale P.M.E., vol. 18, nos 3-4, sous la direction de Louis Raymond P PME1803N

Tous droits de reproduction, de traduction et d'adaptation réservés 
Sonnenfeld, J. (1988), The Hero's Farewell: What Happens When CEOs Retire, New York, Oxford University Press.

Stavrou, E.T. (1998), «A four factor model: a guide to planning next generation involvement in the family firm», Family Business Review, vol. XI, n 2, p. 135-141.

YIN, R.K. (1994), Case Study Research: Design and Method, $2^{\mathrm{e}}$ édition, Thousand Oaks, Cal., Sage Publications.

Revue internationale P.M.E., vol. 18, $\mathrm{n}^{\text {os }} 3-4,2005$

(C) 2005 - Presses de l'Université du Québec

Édifice Le Delta I, 2875, boul. Laurier, bureau 450, Sainte-Foy, Québec G1V 2M2 • Tél.: (418) 657-4399 - www.puq.ca Tiré de: Revue internationale P.M.E., vol. 18, nos 3-4, sous la direction de Louis Raymond • PME1803N Tous droits de reproduction, de traduction et d'adaptation réservés 